\title{
Total Hip Replacement in Patients with Rheumatoid Arthritis: Trends in Incidence and Complication Rates Over 35 Years
}

\author{
Owen Taylor-Williams (D) · Charles A. Inderjeeth • Khalid B. Almutairi • \\ Helen Keen · David B. Preen · Johannes C. Nossent
}

Received: October 9, 2021 / Accepted: December 9, 2021 / Published online: January 8, 2022

(C) The Author(s) 2022

\section{ABSTRACT}

Introduction: Advances in rheumatoid arthritis (RA) management have made disease remission achievable. We evaluated trends in total hip replacement (THR) and postoperative outcomes in patients with RA in Western Australia (WA) over more than three decades.

Methods: This was a retrospective analysis of routinely collected prospective data from a state-wide registry containing longitudinally linked administrative health data based on International Classification of Diseases (ICD) diagnostic and procedural codes. We included

Supplementary Information The online version contains supplementary material available at https:// doi.org/10.1007/s40744-021-00414-9.

O. Taylor-Williams · C. A. Inderjeeth .

K. B. Almutairi · J. C. Nossent ( $\square)$

Rheumatology Group, Medical School, University of

Western Australia, 35 Stirling Highway (M503),

Perth, WA, Australia

e-mail: johannes.nossent@uwa.edu.au

C. A. Inderjeeth $\cdot$ H. Keen $\cdot$ J. C. Nossent

Department of Rheumatology, Sir Charles Gairdner

Hospital, Perth, WA, Australia

H. Keen

Department of Rheumatology, Fiona Stanley

Hospital, Perth, WA, Australia

D. B. Preen

School of Population and Global Health, University

of Western Australia, Perth, WA, Australia patients with two or more diagnostic codes for RA (between 1980 and 2015) and studied THR incidence rates (THR IR) and complication rates (revision, peri-prosthetic fracture, infection, venous thrombosis, and mechanical loosening). Survival rates were estimated by Kaplan-Meier method and predictors analyzed by Cox regression.

Results: We followed 9201 RA patients over 111,625 person-years, during which 1560 patients (16.9\%) underwent THR. From 1985 to 2015, THR IR (per 1000 RA patient-years) decreased from 20.8 (95\% CI 20.1-21.5) to 7.3 (95\% CI 7.2-7.5), and 5-year THR-free survival increased from 84.3 to $95.3 \%$ (1980-2015). Tenyear prosthetic survival was $91.2 \%$. Complication rates in the first 5 years post-THR decreased significantly from 13.1 to $3.7 \%(p<0.001)$. Mechanical complications such as loosening and periprosthetic fracture rates decreased significantly $(>35 \%, P<0.05)$, while infection and revision did not change over the observation period $(p>0.05)$.

Conclusions: Over the last 30 years in RA patients, THR IR and mechanical complication rates decreased significantly, but the medical complication of infection has not changed significantly.

Keywords: Total hip replacement; Rheumatoid arthritis; Incidence; Complications; Diseasemodifying antirheumatic drugs; Epidemiology; Hospital records 


\section{Key Summary Points}

Background: Improved management of rheumatoid arthritis (RA) over the last decades has made disease remission an achievable goal

Hypothesis: The need for total hip replacement (THR) and the rate of postoperative complication rates in RA patients has decreased over the last decades.

Conclusions: In Australian RA patients, THR incidence rates decreased significantly over time and well before the introduction of bDMARDs. Rates for mechanical post-operative complications (aseptic loosening and periprosthetic fracture) decreased, while infection rates remain unchanged.

What was learned: More aggressive RA treatment with csDMARD over the last decades has been associated with a more than 50\% decrease in the need for THR in RA. Whether increased use of bDMARDs further reduces the need for THR and rate of postoperative complications remains to be elucidated.

\section{INTRODUCTION}

Rheumatoid arthritis (RA) is a chronic autoimmune disease where synovial inflammation causes painful swelling of the joints and may erode cartilage and bone [1-3]. Despite best management, RA patients can experience progressive disease and require total hip replacement (THR) $[1,4]$. THR is an option considered on failure of the medical treatment protocol for RA and provides an objective measure of disease severity $[1,2,4]$. Recently, it has become clear that newer medications and management protocols have made low RA disease activity and remission achievable [2, 5-7].
THR incidence rates (THR IR) have been decreasing globally and as of 2010 rates were approximately 7 per 1000 person-years (PY)[8]. However, there is significant heterogeneity between studies and one proposed reason for this is variations in country or health systemspecific factors [8]. This review noted a lack of population-based Australian studies examining THR rates in RA patients [8].

THR aims to relieve pain and improve function in RA patients, but the procedure still carries a risk of adverse outcomes (AO) despite introducing new postoperative protocols, surgical techniques, prosthesis designs, and preventative measures for infections [8-11]. Furthermore, RA patients are reported to have worse post-operative outcomes than osteoarthrosis (OA) patients, which could potentially be influenced by rheumatic disease control $[9,12,13]$, yet there remain relatively few studies examining prosthetic survival and adverse outcomes following THR in RA patients $[8,14,15]$. Given the relative sparsity of data on THR in RA patients, we performed a longitudinal state-wide population-based observational study of THR rates and complications in RA patients in Western Australia (WA) over the period 1980-2015.

\section{METHODS}

\section{Patients}

This observational study used linked administrative health data on patients in the Western Australian Rheumatic Disease Registry (WARDER). The registry, described in detail elsewhere [16], includes all patients in WA with a hospitalbased diagnosis of rheumatic disease between 1980 and 2015. Notably, the registry comprises private and public hospital data but excludes data relating to primary care physicians or pharmaceutical prescriptions. All data in WARDER is coded using the period-relevant version of the International Classification of Diseases (ICD).

Among 18,473 RA patients registered in WARDER, we selected patients with two or more ICD codes for RA using a pre-determined 
algorithm reviewed by the WA Department of Health Clinical Coding group (Table 1). The selection of patients with two or more RA codes ensured a high degree of sensitivity and specificity [17], and did not change the sociodemographic characteristics of the study population (Table S1). Subsequently, 9532 RA patients were identified. Of these patients, 1891 (20.6\%) undergoing THR were identified by relevant ICD codes (Table 1 ). We then excluded patients who had undergone THR before first RA record $(n=331,3.5 \%)$. Information extracted from the remaining $9201 \mathrm{RA}$ patients (1560 undergoing THR) included age at first hospital contact for RA, sex, date of THR, and adjusted Charlson Comorbidity Index (CCI) at first RA contact and at THR. The adjusted CCI is a modification of the well-validated CCI, where higher scores indicate more comorbidities and are associated with worse survival irrespective of diagnosis [18]. Since all patients in this cohort study had RA, and we were interested in how comorbidities, not RA, affected outcomes. We used an adjusted score that removed the rheumatic components of the CCI (a maximum of 1 point was deducted from the score) [18-20]. Using this data, we estimated survival to mortality (from first RA record) and rates of, and time to adverse outcome of revision, peri-prosthetic fracture, infection, VTE, and mechanical loosening using further ICD codes (Table 1).

Ethics approval for this project was obtained from the Human Research Ethics Committee at the WA Department of Health (HREC 2016.24).

\section{Statistical methods}

Complication rates and incidence rates were compared using the Pearson's Chi-square test. Mann-Whitney $U$ test was used for pairwise comparison of continuous variables (gender, age, CCI). For statistical analysis of rates, patients were categorized into age groups $(<50$, $50-65,65-80$, and $80+$ years), year of first RA record (5-year periods from 1980 to 2015), and year of THR (5-year periods from 1980-2015). Incidence rates (IR) were calculated by dividing the number of THR in each time period by the total number of PY contributed by at risk
Table 1 ICD codes used for each concept, backward and forward mapped into the various versions of ICD codes used during our study 1980-2015

\begin{tabular}{|c|c|c|c|}
\hline Concept & ICD 10 & $\begin{array}{l}\text { ICD } 9 \text { (used } \\
1 / 1 / \\
1988-30 / 6 / \\
1999)\end{array}$ & $\begin{array}{l}\text { ICD } 9 \text { (used } \\
\text { prior to } \\
31 / 12 / \\
1987)\end{array}$ \\
\hline \multirow{3}{*}{$\begin{array}{l}\text { Hip } \\
\text { replacement }\end{array}$} & $49,318-00$ & 81.51 & 58.15 \\
\hline & $49,319-00$ & V43.64 & V43.6 \\
\hline & Z96.64 & & \\
\hline \multirow[t]{8}{*}{ Revision } & $49,312-00$ & 80.95 & 58.16 \\
\hline & $49,324-00$ & 80.05 & \\
\hline & $49,327-00$ & 81.53 & \\
\hline & $49,330-00$ & & \\
\hline & $49,333-00$ & & \\
\hline & 49,339-00 & & \\
\hline & $49,342-00$ & & \\
\hline & $49,345-00$ & & \\
\hline \multirow[t]{3}{*}{ Dislocation } & T84.0 & 996.4 & 996.4 \\
\hline & $47,048-00$ & 79.75 & 82.09 \\
\hline & $47,051-00$ & 79.85 & 57.96 \\
\hline Loosening & T84.0 & 996.4 & \\
\hline \multirow{13}{*}{$\begin{array}{l}\text { Periprosthetic } \\
\text { fracture }\end{array}$} & M96.6 & 996.78 & \\
\hline & & 996.7 & \\
\hline & $47,498-00$ & 78.55 & 57.87 \\
\hline & $47,501-00$ & 78.59 & 57.9 \\
\hline & $47,525-00$ & 79.05 & 57.91 \\
\hline & $47,525-01$ & 79.15 & 57.92 \\
\hline & $47,528-00$ & 79.25 & 57.93 \\
\hline & $47,528-01$ & 79.35 & 57.94 \\
\hline & $47,531-00$ & 79.39 & 82.04 \\
\hline & $47,534-00$ & 79.45 & \\
\hline & $47,537-00$ & 79.55 & \\
\hline & $47,516-01$ & & \\
\hline & $47,519-00$ & & \\
\hline Infection & T84.5 & 996.66 & 996.6 \\
\hline
\end{tabular}


Table 1 continued

\begin{tabular}{|c|c|c|c|}
\hline Concept & ICD 10 & $\begin{array}{l}\text { ICD } 9 \text { (used } \\
1 / 1 / \\
1988-30 / 6 / \\
1999)\end{array}$ & $\begin{array}{l}\text { ICD 9 (used } \\
\text { prior to } \\
31 / 12 / \\
1987 \text { ) }\end{array}$ \\
\hline \multirow[t]{2}{*}{ VTE } & T84.8 & 996.77 & 996.7 \\
\hline & T84.82 & 996.78 & \\
\hline
\end{tabular}

patients (in that time period) and presented per 1000 PY. At risk was defined as having an RA code recorded in the time period of interest or prior, and not having received THR or died. Significant differences in IR were tested for the overall study (where the expected rates derived from the 1980s group) and between incremental periods (where the predicted rate is derived from the previous time period). $P$ values less than 0.05 were interpreted as indicating a significant change in IR had occurred. THR-free survival for the entire RA cohort was calculated as the time (in days) from the first recorded RA diagnostic code to the end of study (2015) or patient death date, with the first THR code as the event of interest. Patient survival after THR was calculated as the time from THR to the end of study or patient death, while AO-free survival times was calculated as the time from THR to the end of study or patient death, with $\mathrm{AO}$ as the event of interest. The frequency of each adverse outcome was also calculated, with events censored at the maximum follow-up times of 6 months, 5, 10, and 15 years postTHR. However, VTE was only followed to 6 months, as longer time periods were deemed unlikely to be related to THR [21]. Maximum follow-up times were selected to allow an examination of both short- and long-term complication rates [5, 22-26].

Survival rates were calculated using Kaplan-Meier life survival tables and compared using the log-rank Mantel-Cox test. Hazard ratios (HR) were estimated using Cox regression analysis. In Cox regression models, the covariates of age at first contact, age at THR, sex, CCI, year of RA diagnosis, and year of THR were adjusted. This analysis was conducted using Statistical Package for the Social Sciences software (IBM SPSS Statistics for Windows, Version 27, IBM Corp., Armonk, NY, USA), with additional analysis in Microsoft Excel 2019 (Microsoft, Richmond WA, USA).

\section{RESULTS}

\section{Demographics}

There were proportionally more females in the THR cohort compared to the non-THR patients $(7.9 \%, p<0.001)$ (Table 2 and S2) but age at first RA contact did not differ significantly between THR vs. non-THR patients $(p>0.2)$. Age at first hospital contact decreased in the cohort of patients later requiring THR from 63.6 years to 55.1 years $(p<0.001)$, but not for the full RA cohort (mean age 59.4, $p>0.1$ ) (Table 2). Women had a lower CCI at first RA hospital contact than men $(p<0.01)$ and THR patients had lower CCI scores than non-THR patients at first RA contact $(p<0.001)$ (Table S1). CCI scores at time of THR did not change significantly throughout the study $(p>0.9)$ (Table 2 and Table S2).

\section{Total hip replacement-free and patient survival}

In the full RA cohort, THR-free survival increased throughout the study (5-year survival increased from $84.3 \%$ to $95.3 \%$ ) (Table 3). Risk factors for progressing to THR were increasing age at first RA contact and female sex (HR $>1.2$, $p<0.001)$, while later study entry and higher CCI at first RA contact reduced risk of THR (HR $<0.90, p<0.001)$ (Table 4). After controlling for age at RA diagnosis, CCI at RA diagnosis, and sex, progressing to THR did not significantly influence mortality rates in RA patients $(p=0.5, \mathrm{HR}=1.03)$ (Table S3 and S4). Patient survival was influenced by CCI $(p<0.001$, HR 1.05) and age at first RA contact $(p<0.001$, HR 2.27) (Table S4). 


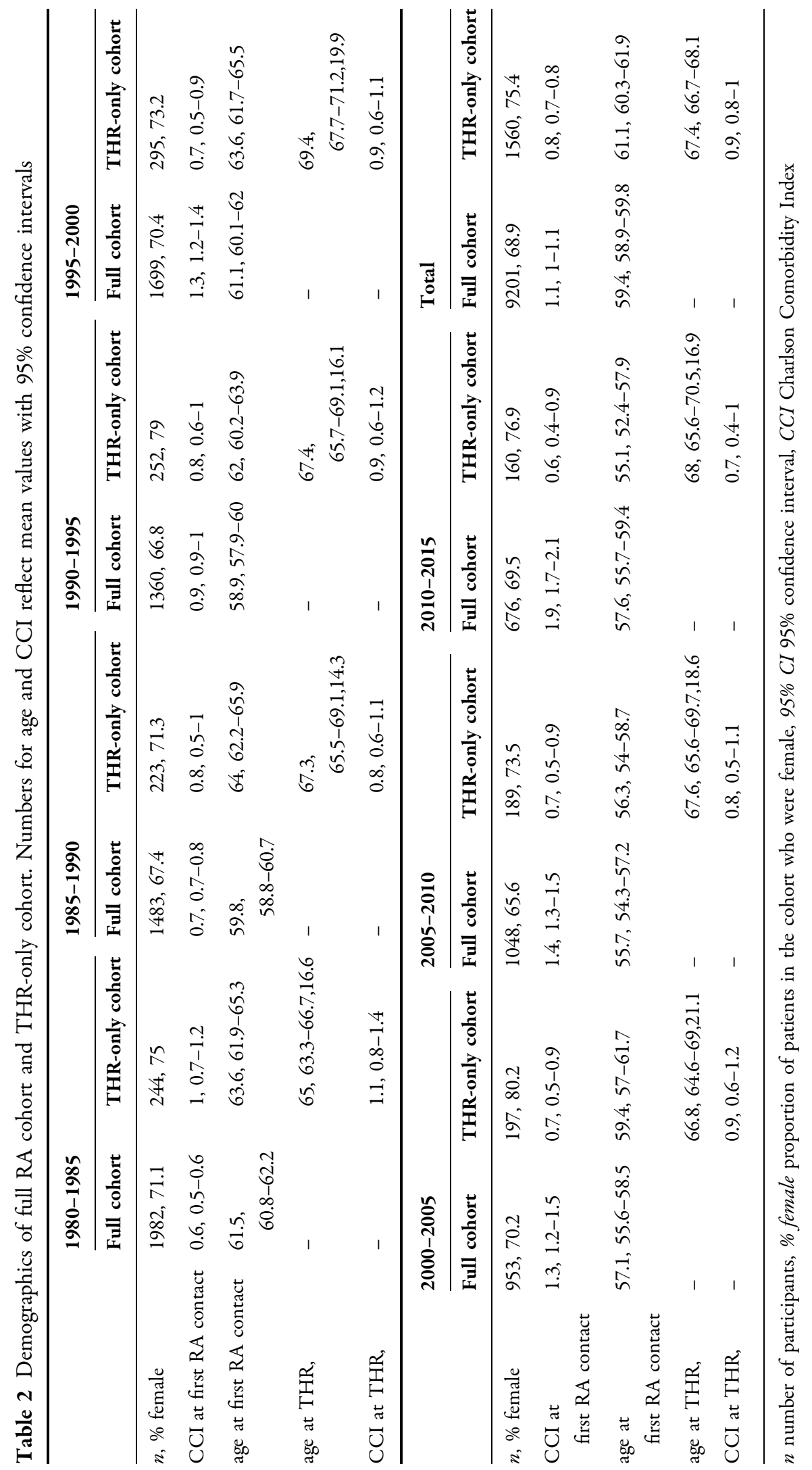




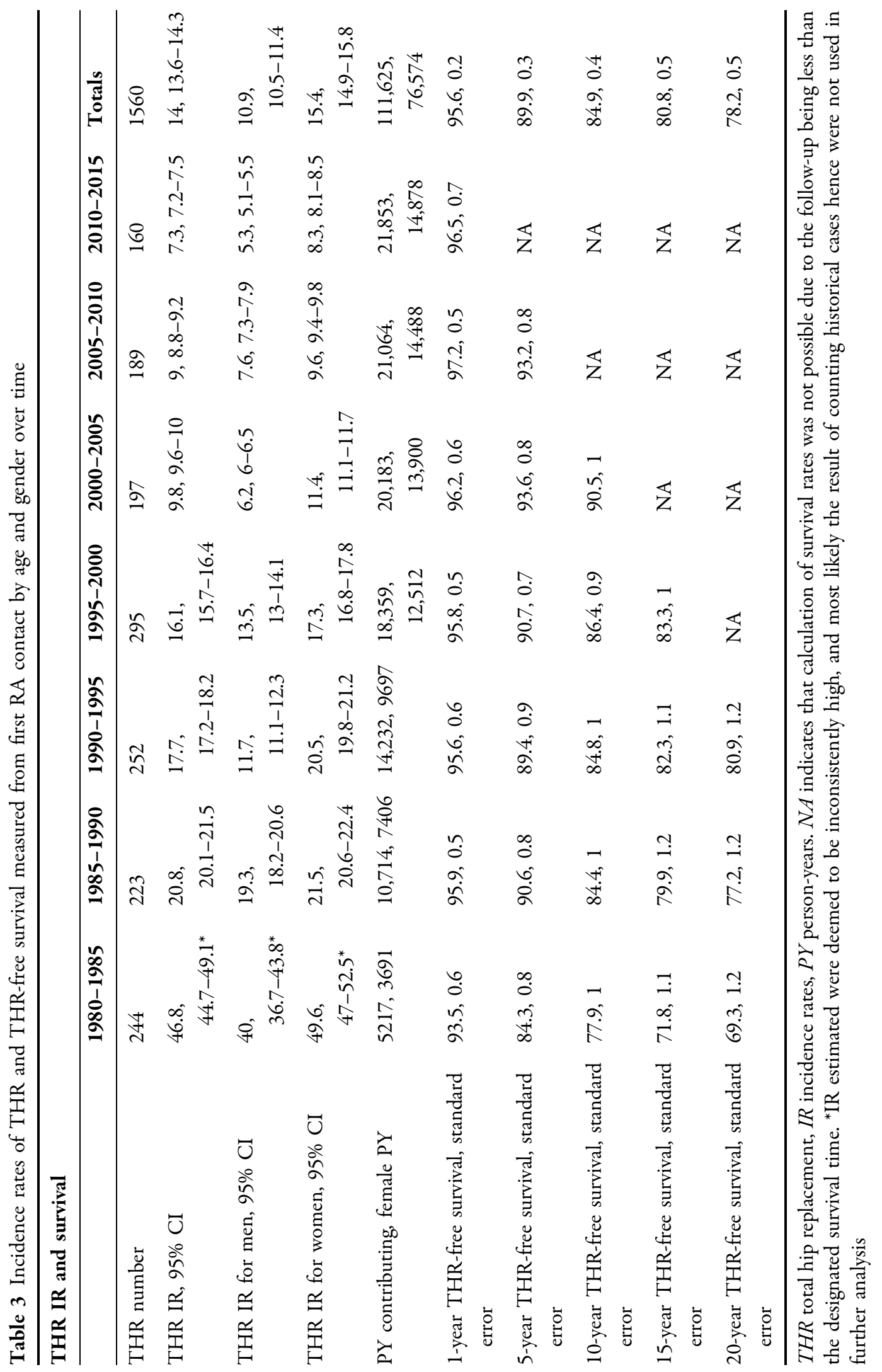


Table 4 Covariates used in the Cox regression analysis for survival from first RA contact to THR

\begin{tabular}{llll}
\hline & $\begin{array}{l}\text { Significance } \\
(\boldsymbol{p} \text { value })\end{array}$ & HR & $\mathbf{9 5 \%}$ CI \\
\hline $\begin{array}{l}\text { Gender } \\
\text { Age at first RA }\end{array}$ & $<0.001$ & 1.370 & $1.22-1.54$ \\
$\begin{array}{c}\text { contact } \\
\text { Year of first RA } \\
\text { contact }\end{array}$ & $<0.001$ & 1.290 & $1.22-1.36$ \\
$\begin{array}{l}\text { CCI at first RA } \\
\text { contact }\end{array}$ & $<0.001$ & 0.848 & $0.82-0.88$ \\
\hline
\end{tabular}

$H R$ hazard ratio; 95\% CI 95\% confidence interval, $C C I$ Charlson Comorbidity Index

\section{Trend in total hip replacement incidence}

THR IR decreased significantly $(p<0.01)$, from 20.8/ 1000 PY (95\% CI 20.1-21.5) in 1985-1990, to $7.3 / 1000 P Y$ (95\% CI 7.2-7.5) in 2010-2015, with the greatest decrease seen from 1995-2000 to 2000-2005 (Fig. 1 and Table 3). Trends were similar in male and female patients, with a slightly higher THR IR in females (Fig. 1).

\section{Adverse outcomes}

At 5,10 , and 15 years post-THR, the only complications to decrease significantly throughout our study were periprosthetic fracture and loosening, decreasing by in excess of $35 \%$ $(p<0.05)$. Infection rates and dislocation did not change significantly $(p>0.05)$. Table 5 and Figs. 2 and 3.

Five-year AO rates, for all outcomes combined, averaged $8.0 \%$, decreasing from $13.1 \%$ to $2.5 \%$ from 1980 to 2010, respectively $(p<0.001)$ (Table 5). A similar trend was observed for adverse outcomes at 15 years post THR which decreased from $21.1 \%$ to $12.2 \%$ from 1985 to $2000(p<0.001)$ (Table 5).

\section{Dislocation}

Dislocation rates decreased over the duration of our study. Five-year dislocation rates averaged $6.09 \%$ and decreased from 7.8 to $3.7 \%$ (1980-2010, $p=0.08$ ) (Table 5 and Fig. 2). Similarly, 15-year dislocation rates decreased from 20.9 to $14.6 \%(1980-2000, p=0.054)$ (Table 5 and Fig. 3). The probability of dislocation-free survival reduced $(p<0.01)$ with a later year of undergoing THR (HR 1.18, 95\% CI 1.05-1.34) Sex, CCI, and age were not significantly associated with dislocation in any model $(p>0.05)$ (Table S5).

\section{Loosening}

Loosening rates averaged $14.3 \%$ at 15 years of follow-up and decreased significantly from 20.5 to $12.2 \%, 1980-2000(p<0.01)$ (Table 5 and Fig. 3). A similar, but non-significant, decrease was observed at 5 and 10 years of follow-up. From Cox regression models, year of THR was the only variable associated with loosening-free survival. The subsequent year of THR conferred a reduced survival (HR 1.16, 95\% CI 1.02-1.32);

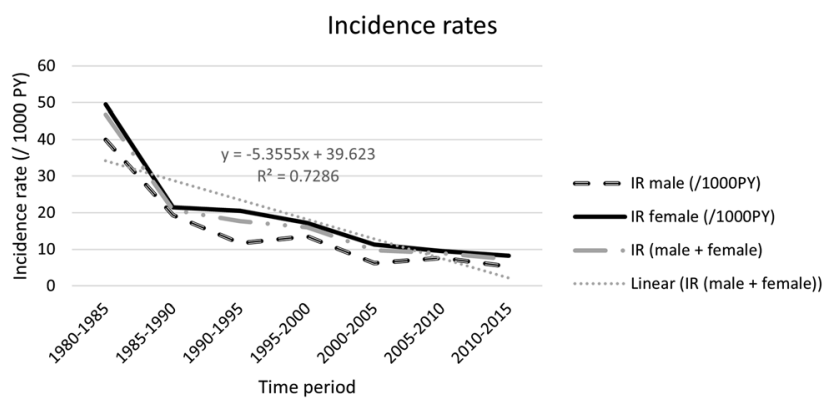

Fig. 1 THR incidence rates in RA patients by gender during 5-year intervals in study period 1980-2015 


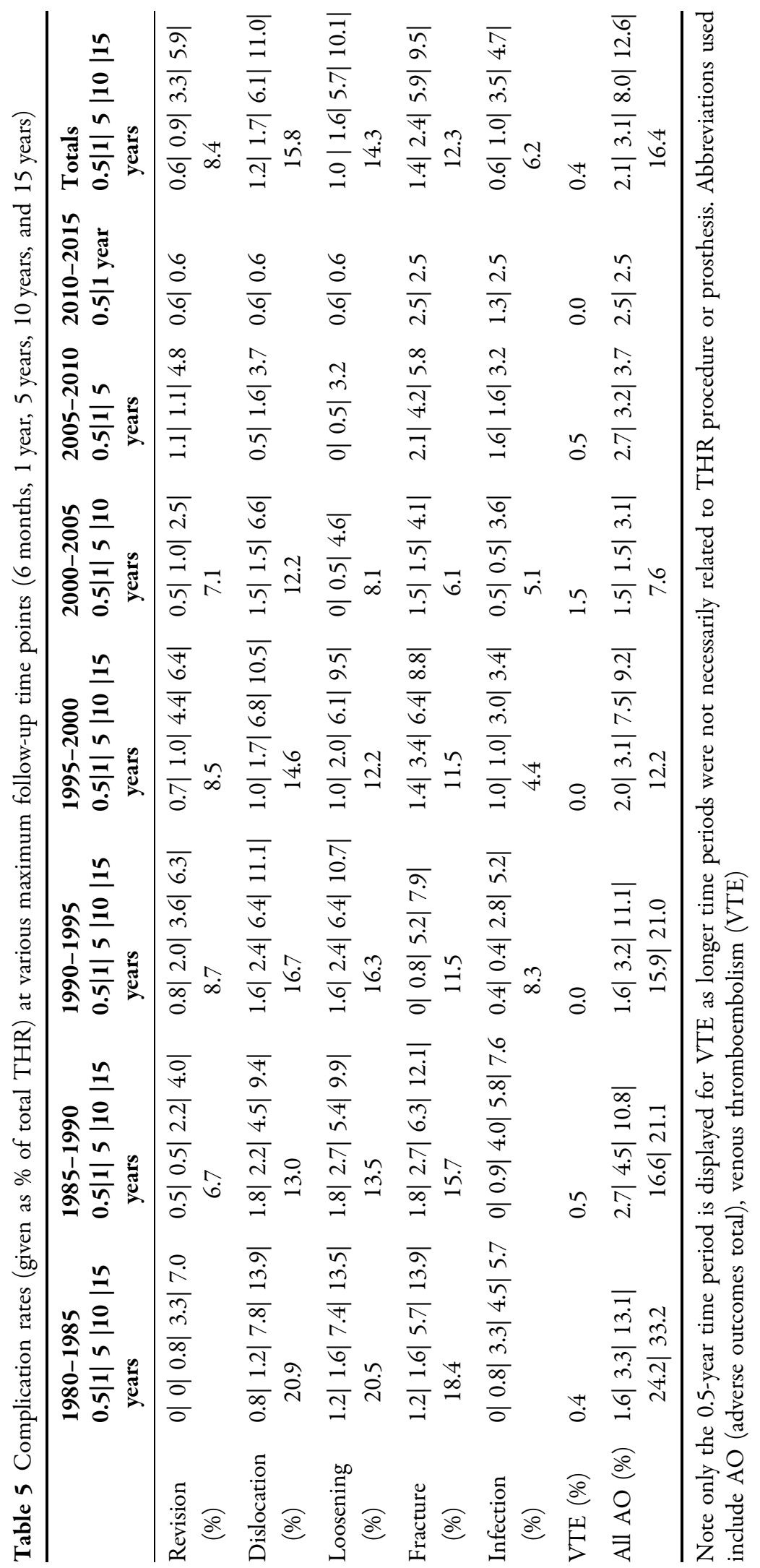



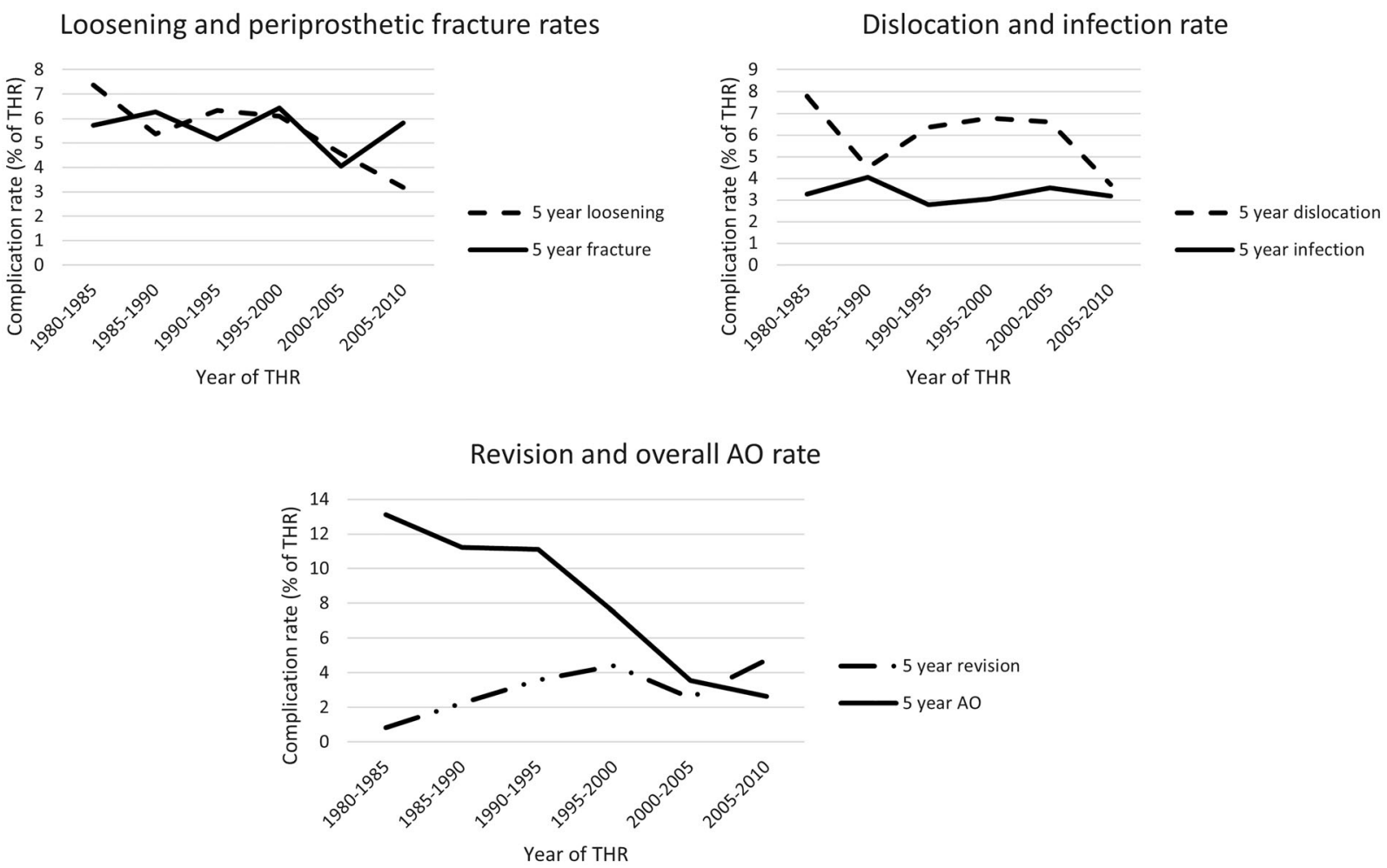

Fig. 2 Complication rates (as percentage of THR performed) at a maximum intervals of 5-year follow-up in study period 1980-2010

while sex, CCI, and age were not significantly associated with loosening $(p>0.05)$ (Table S5).

\section{Revision}

Revision rates at 5, 10, and 15 years did not decrease significantly over the duration of the study. 10-year revision rates increased from 3.3 to $7.1 \%$ (1980-2005); however, this was not significant $(p=0.06)$ (Table 5 , Figs. 2,3$)$. The subsequent year of THR conferred a reduced survival rate (HR 1.34, 95\% CI 1.17-1.62), while sex, CCI, and age were not significantly associated with this outcome $(p>0.05)$ (Table S5).

\section{VTE}

A total of eight VTE events were identified in the first 6 months post-THR (0.5\%)(Table 5). Unfortunately, this meant there was an insufficient number to carry out further analysis (stratification, survival, or trend over time).

\section{Infection}

Infection rates did not change significantly throughout our study $(p>0.4)$ (Table 5, Figs. 2, 3 ). Additionally, no variables were significantly associated with infection-free survival. (Table S5).

\section{Periprosthetic fracture}

Periprosthetic fracture rates decreased significantly, with 10-year fracture rates decreasing from 13.9 to $6.1 \%(1980-2005), p=0.007$ (Table 5, Fig. 3). Older patients at first RA contact had worse survival (HR 1.62, CI 1.33-2.30); while sex, year of THR, CCI, and age at THR did not prove significant associated with periprosthetic fracture rates $(p>0.05)$. (Table S5). 

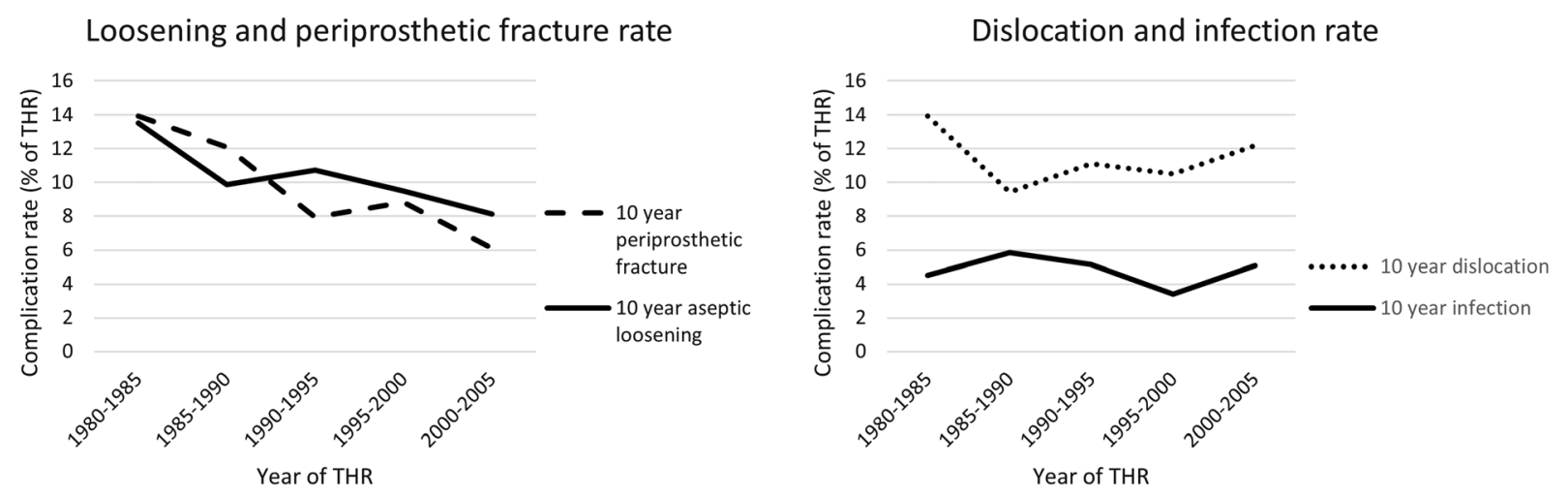

Revision and overall $\mathrm{AO}$ rate

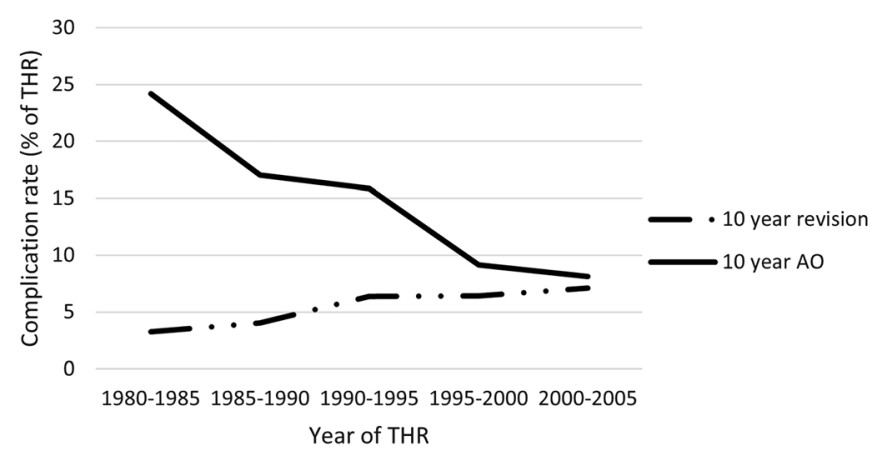

Fig. 3 Complication rates after maximum of 10-year follow-up in study period 1980-2005

\section{DISCUSSION}

We found that THR incidence for RA patients decreased over time, with the most significant change occurring around 2000 following a decade where Methotrexate therapy became anchored as first-line therapy for RA. The change in THR IR noted around 1985, is likely a result of the prevalent pool effect due to inclusion of patients with RA before 1980. RA patients progressing to THR encountered the hospital system at a younger age, had fewer comorbidities, and were more often female. THR did not influence patient survival, postoperative rates of periprosthetic fracture, and loosening decreased, but dislocation, infection, and revision rates did not change significantly over time.

THR IR for RA patients decreased significantly, which is in clear contrast to the THR IR for osteoarthrosis (OA) patients and the general population which continues to increase (approximately 50/100,000 patients in 1994 to $133 / 100,000$ patients in 2017, in the Australian general population) [27-33]. The opposing trends for THR in RA and OA populations suggest an RA-specific cause for the reduction in THR IR, as opposed to a general reduction in THR rates (for OA and RA), which would have suggested a less RA-specific cause such as fewer THR being available. The role of RA management is further supported by our finding that later years of entry to the RA cohort reduced the HR for THR, consistent with other studies [8]. With RA and OA patients now undergoing THR at similar ages (65-69 years old), it would appear RA disease management has been successful in reducing RA joint destructive potential [27, 32, 34, 35].

THR IR reduced significantly from 1995-2000 to 2000-2005, which is just prior to the widespread use of bDMARDs, in a period 
where increased use of csDMARD and shortening time to treatment occurred in the decade prior [36-41]. We found further support for the importance of early, aggressive antirheumatic therapy, as older patients at their first hospital contact for RA were at a higher risk of THR. If patients have an approximately constant age of onset, this elevated risk of THR in older patients could suggest a higher accumulation of joint damage due to slower time to treatment and worse RA control before THR. Alternatively, this could be the consequence of reasonable RA disease control in the community resulting in patients coming in contact with the hospital system, at older ages, due to age-related OA degeneration of the hip, as opposed to RA destruction. A further explanation for the significant decrease in THR IR around 2000 is a change from ICD9 to ICD10 coding in 1999.

An alternative explanation for reducing THR IR could be that fewer RA patients are deemed medically fit for surgery. One way of assessing medical fitness is via the CCI score [42]. In our study, patients with a higher CCI at first RA contact were less likely to receive a THR, suggesting there may be an element of surgical selection for more medically fit patients. Potentially due to surgical selection, the CCI at first RA contact for the THR cohort has remained unchanged, while the CCI at first RA contact for the entire RA cohort has continued to increase. Other studies have suggested THR is protective against mortality due to surgical selection identifying healthier patients [43]. However, after controlling for age at RA index date, CCI at first RA contact, and sex, our survival analysis suggests THR does not influence mortality rates in RA patients $(p>0.05)$. Survival was only reduced in patients with higher CCI and older age at RA diagnosis.

In summary, decreasing THR IR appear to be the result of a RA-specific factors coincide with the introduction of csDMARDs and the concept of the window of therapeutic opportunity, and occurred before the mainstream introduction of bDMARD.

\section{THR complications}

RA patients undergoing THR now have good postoperative outcomes, comparable to $\mathrm{OA}$ patients, with 1- and 5-year complication rates averaging under $3 \%$ and $8 \%$, respectively $[44,45]$. Declining 5-year postoperative complication rates from $8 \%$ to under $4 \%$ suggest a general improvement in the management of RA THR patients. Possible explanations for decreasing complication rates include increasing surgical THR volume in the general population, changes to surgical technique, and surgical selection of fitter patients [28, 46-48]. We found higher CCI increases HR for THR, THR patients have lower CCI (vs. non-THR), and CCI in THR patients has remained constant. This may suggest that surgical selection has prevented increasing complication rates, but has not resulted in decreasing rates of adverse outcomes.

Rates for individual complications did not decline equally. Mechanical complications potentially associated with the underlying RA disease activity (loosening and periprosthetic fracture) changed most significantly $[35,49-51]$, as opposed to infection, a nonmechanical complications.

Infection rates in this study are consistent with international and Australian rates for OA and RA populations and have not changed significantly over time $[8,52,53]$. This could suggest new infection control protocols have not affected postoperative complication rates to a large extent. Alternatively, changes to RA management, such as the increased use of DMARDs and bDMARDs, may have offset improvements resulting from modifications to infection control protocols [54-57]. However, the fact that infection rates are similar compared to the Australian OA and general population undergoing THR provides reassurance that the current use of antirheumatic drugs, does not place RA patients at an elevated risk of postoperative infection.

Complications potentially related to the underlying RA pathology and its management (such as corticosteroids), including aseptic loosening and periprosthetic fracture, have decreased over time in line with other studies 
$[35,49-51]$. We note periprosthetic fracture rates found in this study, contrast against the trend observed in OA patients, potentially suggesting improvements in RA disease management have at least partially improved postoperative outcomes [58-61]. While various studies report significantly different rates of periprosthetic fracture, due to population heterogeneity, it appears periprosthetic fracture rates are increasing [23, 62-64]. Lindahl et al. in a Swedish registry study from 1979-2000 found a nearly threefold increase in incidence from $0.045 \%$ to $0.13 \%$ [63], while Miettinen et al. found a similar threefold increase from $1.6 /$ 1000 PY to 4.5/ 1000 PY [65]. Furthermore, aseptic loosening rates appear to have decreased faster in RA patients, with RA and OA patients now having comparable loosening rates at $8 \%$ [66-71]. However, as these comparisons are made with similar studies and not directly with a comparative OA cohort, and a significant number of advances have occurred surrounding THR surgery, alternative explanations must be considered. Potential alternative explanations for the demonstrated improvements in postoperative outcomes include changes to prosthesis materials, patient optimization, surgical approach, and post-operative rehabilitation [51, 72-74].

Revision rates were low, under $1 \%$ at 1 year and $4 \%$ at 5 years, which is consistent with international rates [8] and remained constant across the duration of our study, suggesting that there has been improvement in treating other complications but not in preventing complications progressing to revision. Specifically, it appears that the increasing use of cementless fixation and newer prosthesis designs have not improved revision rates [52]. However, OA patients in the Australian Orthopaedic Association National Joint Replacement Registry (AOANJRR) have better prosthesis survival rates compared to RA [35]. Hence, it appears that there is still room for improvement regarding the surgical management of RA patients.

\section{Strengths and limitations}

This large population-based RA cohort, with detailed demographic data, allowed us to consider whether the global changes in RA management, over time have improved severe disease outcomes in a non-selected population with statistical precision and high external validity. The long follow-up time allowed us to analyze changes to late complication rates, not able to be examined in many other studies. Lastly, the fact that diagnostic and procedural codes, which are based on a physician's diagnosis and transcribed by trained clinical coders, have been verified in previous studies [75], provides strong reassurance to the accuracy of codes used in this study.

Limitations result from the reliance solely on hospital-based administrative data and lack of a comparator group. The use of hospital-based administrative health data means more detailed clinical data, such as imaging, laboratory data, and pharmacological therapy were unavailable. With regards to the lack of a comparable OA cohort, we have tried as much as possible to make comparisons with studies and registries published within the region to minimize the effect of regional variations on our conclusions. A further limitation is the potential prevalent pool risk in the early years of study where prevalent cases of THR might have been incorrectly identified as incident cases, but we reduced this risk by using a 5-year wash-out period from 1980 to 1984 . Regarding the use of the ICD code, each revision of the ICD system results in imperfect backward mapping; hence complications rates, particularly in the early years of study, are based on less detailed coding.

\section{CONCLUSIONS}

RA patients have become much less likely to require a THR and overall experience better postoperative outcomes. This likely results from the global trend of earlier and more effective antirheumatic drug therapy resulting in better disease control and lower the risk of joint damage. Whether the introduction of bDMARD therapy leads to further improvements in THR 
incidence and outcomes will require further long-term study.

\section{ACKNOWLEDGEMENTS}

The authors thank the staff at the Western Australian Data Linkage Branch and the Data Custodians of the Hospital Morbidity Data Collection, Emergency Department Data Collection and the State and Territory Registries of Births, Deaths and Marriages.

Funding. Owen Taylor-Williams was supported by the Cottesloe Women's Service Guild Seaman Scholarly Plus Award in Arthritis Research at the University of Western Australia. KA was supported by an Australian Government Research Training Program PhD Scholarship at the University of Western Australia and the Australian Rheumatology Association WA Research Fellowship Award. WARDER was supported by an unrestricted grant by The Arthritis Foundation of WA to JCN. No Rapid Service Fee was received by the journal for the publication of this article.

Authorship. All named authors meet the International Committee of Medical Journal Editors (ICMJE) criteria for authorship for this article, take responsibility for the integrity of the work as a whole, and have given their approval for this version to be published.

Author Contributions. Study concept and design: OTW, JN, CAI; Acquisition of data: OTW, KA; Analysis of data: OTW; Writing and critically commenting of the manuscript: All authors.

Disclosure. Owen Taylor-Williams, Charles A Inderjeeth, Khalid B Almutairi, Helen Keen, David B Preen, Johannes Nossent all have nothing to disclose.

Compliance with Ethical Guidelines. Ethics for this project was approved by the Human Research Ethics Committee at the WA Department of Health (HREC 2016.24). We confirm that it was completed in accordance with the Helsinki Declaration of 1964.

Data Availability. All data generated or analyzed during this study are included in this published article/as supplementary information files.

Open Access. This article is licensed under a Creative Commons Attribution-NonCommercial 4.0 International License, which permits any non-commercial use, sharing, adaptation, distribution and reproduction in any medium or format, as long as you give appropriate credit to the original author(s) and the source, provide a link to the Creative Commons licence, and indicate if changes were made. The images or other third party material in this article are included in the article's Creative Commons licence, unless indicated otherwise in a credit line to the material. If material is not included in the article's Creative Commons licence and your intended use is not permitted by statutory regulation or exceeds the permitted use, you will need to obtain permission directly from the copyright holder. To view a copy of this licence, visit http://creativecommons.org/licenses/by$\mathrm{nc} / 4.0 /$.

\section{REFERENCES}

1. Nikiphorou E, Konan S, MacGregor AJ, Haddad FS, Young A. The surgical treatment of rheumatoid arthritis. Bone Jt J 2014;96-B(10):1287-9.

2. Nikiphorou E, Norton S, Young A, et al. Association between rheumatoid arthritis disease activity, progression of functional limitation and long-term risk of orthopaedic surgery: combined analysis of two prospective cohorts supports EULAR treat to target DAS thresholds. Ann Rheum Dis. 2016;75(12): 2080-6.

3. Salama A, Hamer AJ. Degenerative and rheumatoid arthritis (including joint replacement). Surg Infect (Larchmt). 2007;25(4):160-5.

4. Mancuso CA, Ranawat CS, Esdaile JM, Johanson NA, Charlson ME. Indications for total hip and total knee arthroplasties: results of orthopaedic surveys. J Arthroplasty. 1996;11(1):34-46. 
5. Learmonth ID, Young C, Rorabeck C. The operation of the century: total hip replacement. Lancet (London, England). 2007;370(9597):1508-19.

6. Wolfe F, Zwillich SH. The long-term outcomes of rheumatoid arthritis: a 23-year prospective, longitudinal study of total joint replacement and its predictors in 1,600 patients with rheumatoid arthritis. Arthritis Rheum. 1998;41(6):1072-82.

7. Raza K, Filer A. The therapeutic window of opportunity in rheumatoid arthritis: does it ever close? : BMJ Publishing Group Ltd; 2015.

8. Taylor-Williams $\mathrm{O}$, Nossent J, Inderjeeth CA. Incidence and complication rates for total hip arthroplasty in rheumatoid arthritis: a systematic review and meta-analysis across four decades. Rheumatol Therap. 2020;7(4):685-702.

9. Ravi B, Escott B, Shah PS, et al. A systematic review and meta-analysis comparing complications following total joint arthroplasty for rheumatoid arthritis versus for osteoarthritis. Arthritis Rheum. 2012;64(12):3839-49.

10. Bozic KJ, Kurtz SM, Lau E, et al. The epidemiology of revision total hip arthroplasty in the United States. JBJS. 2009;91(1):128-33.

11. Karachalios T, Komnos G, Koutalos A. Total hip arthroplasty: Survival and modes of failure. EFORT Open Rev. 2018;3(5):232-9.

12. Rud-Sørensen C, Pedersen AB, Johnsen SP, Riis AH, Overgaard S. Survival of primary total hip arthroplasty in rheumatoid arthritis patients: findings in 1,661 arthroplasties in 1,395 patients from the Danish Hip Arthroplasty Registry. Acta Orthop. 2010;81(1):60-5.

13. Ravi B, Croxford R, Hollands $\mathrm{S}$, et al. Increased risk of complications following total joint arthroplasty in patients with rheumatoid arthritis. Arthritis Rheumatol. 2014;66(2):254-63.

14. Burn E, Edwards CJ, Murray DW, et al. The impact of rheumatoid arthritis on the risk of adverse events following joint replacement: a real-world cohort study. Clin Epidemiol. 2018;10:697-704.

15. Clement ND, Breusch SJ, Biant LC. Lower limb joint replacement in rheumatoid arthritis. J Orthop Surg Res. 2012;7(1):27.

16. Ognjenovic $\mathrm{M}$, Raymond $\mathrm{WD}$, Inderjeeth CA, et al. The risk and consequences of vertebral fracture in patients with ankylosing spondylitis: a populationbased data linkage study. J Rheumatol. 2020;47(11): $1629-36$.
17. Almutairi K, Inderjeeth $\mathrm{C}$, Preen $\mathrm{DB}$, et al. The accuracy of administrative health data for identifying patients with rheumatoid arthritis: a retrospective validation study using medical records in Western Australia. Rheumatol Int. 2021;41(4): 741-50.

18. Austin SR, Wong YN, Uzzo RG, Beck JR, Egleston BL. Why summary comorbidity measures such as the Charlson comorbidity index and Elixhauser score work. Med Care. 2015;53(9):e65-72.

19. Norton S, Koduri G, Nikiphorou E, et al. A study of baseline prevalence and cumulative incidence of comorbidity and extra-articular manifestations in RA and their impact on outcome. Rheumatology. 2012;52(1):99-110.

20. Lee S, Chang YJ, Cho H. Impact of comorbidity assessment methods to predict non-cancer mortality risk in cancer patients: a retrospective observational study using the National Health Insurance Service claims-based data in Korea. BMC Med Res Methodol. 2021;21(1):66.

21. Bjørnarå BT, Gudmundsen TE, Dahl OE. Frequency and timing of clinical venous thromboembolism after major joint surgery. J Bone Joint Surg Br. 2006;88(3):386-91.

22. Kunutsor SK, Barrett MC, Beswick AD, et al. Risk factors for dislocation after primary total hip replacement: a systematic review and meta-analysis of 125 studies involving approximately five million hip replacements. Lancet Rheumatol. 2019;1(2): e111-21.

23. Berry DJ, Harmsen WS, Cabanela ME, Morrey BF. Twenty-five-year survivorship of two thousand consecutive primary Charnley total hip replacements: factors affecting survivorship of acetabular and femoral components. J Bone Joint Surg. 2002;84(2):171-7.

24. Ong KL, Kurtz SM, Lau E, et al. Prosthetic Joint Infection Risk After Total Hip Arthroplasty in the Medicare Population. The Journal of Arthroplasty. 2009;24(6, Supplement):105-9.

25. Cook RE, Jenkins PJ, Walmsley PJ, Patton JT, Robinson CM. Risk factors for periprosthetic fractures of the hip: a survivorship analysis. Clin Orthop Relat Res. 2008;466(7):1652-6.

26. Sidler-Maier CC, Waddell JP. Incidence and predisposing factors of periprosthetic proximal femoral fractures: a literature review. Int Orthop. 2015;39(9):1673-82.

27. Network AM. NSW Evidence Review Preoperative, Perioperative and Postoperative Care of Elective 
Primary Total Hip and Knee Replacement. In: Innovation AfC, editor. 2012.

28. Elective Joint Replacement Service Model of Care. In: Department of Public Health WA, editor. 2010.

29. Manning L, Davis JS, Robinson O, et al. High prevalence of older Australians with one or more joint replacements: estimating the population at risk for late complications of arthroplasty. ANZ J Surg. 2020;90(5):846-50.

30. Mak JCS, Fransen M, Jennings M, et al. Evidencebased review for patients undergoing elective hip and knee replacement. ANZ J Surg. 2014;84(1-2): $17-24$.

31. Health AIo, Welfare. Osteoarthritis. Canberra: AIHW; 2020.

32. Australian Orthopaedic Association. National Joint Replacement Registry. Hip and Knee Arthroplasty annual report. (2000). https://aoanjrr.sahmri.com/ annual-reports.

33. Wells VM, Hearn TC, McCaul KA, et al. Changing incidence of primary total hip arthroplasty and total knee arthroplasty for primary osteoarthritis. J Arthroplasty. 2002;17(3):267-73.

34. Young BL, Watson SL, Perez JL, et al. Trends in joint replacement surgery in patients with rheumatoid arthritis. J Rheumatol. 2018;45(2):158-64.

35. Australian Orthopaedic Association. National Joint Replacement Registry. Hip and Knee Arthroplasty annual report. (2020). https://aoanjrr.sahmri.com/ annual-reports.

36. Steffen A, Holstiege J, Klimke K, Akmatov MK, Bätzing J. Patterns of the initiation of diseasemodifying antirheumatic drugs in incident rheumatoid arthritis: a German perspective based on nationwide ambulatory drug prescription data. Rheumatol Int. 2018;38(11):2111-20.

37. Espinoza F, Fabre S, Pers Y-M. Remission-induction therapies for early rheumatoid arthritis: evidence to date and clinical implications. Therap Adv Musculoskelet Dis. 2016;8(4):107-18.

38. Kimsey L, Weissman JS, Patel A, et al. Delay in initiation of DMARD or anti-inflammatory therapy in patients newly diagnosed with rheumatoid arthritis: an analysis of United States Military Health System TRICARE beneficiaries. Semin Arthritis Rheum. 2019;48(5):821-7.

39. Singh JA, Saag KG, Bridges SL Jr, et al. 2015 American College of Rheumatology guideline for the treatment of rheumatoid arthritis. Arthritis Rheumatol. 2016;68(1):1-26.
40. American College of Rheumatology Subcommittee on Rheumatoid Arthritis Guidelines. Guidelines for the management of rheumatoid arthritis: 2002 Update. Arthritis \& Rheumatism. 2002;46(2): 328-46.

41. Therapeutic Goods Administration. AUSTRALIAN PRODUCT INFORMATION - DBL Methotrexate Injection (Methotrexate). In: Health Do, editor. 2020.

42. Voskuijl T, Hageman M, Ring D. Higher Charlson Comorbidity Index Scores are associated with readmission after orthopaedic surgery. Clin Orthop Relat Res. 2014;472(5):1638-44.

43. Barrett J, Losina E, Baron JA, et al. Survival following total hip replacement. JBJS. 2005;87(9): 1965-71.

44. Aggarwal VK, Elbuluk A, Dundon J, et al. Surgical approach significantly affects the complication rates associated with total hip arthroplasty. Bone Joint J 2019;101-b(6):646-51.

45. Ferguson RJ, Palmer AJR, Taylor A, et al. Hip replacement. The Lancet. 2018;392(10158): 1662-71.

46. Charnley J. Present status of total hip replacement. Ann Rheum Dis. 1971;30(6):560-4.

47. Katz JN, Losina E, Barrett J, et al. Association between hospital and surgeon procedure volume and outcomes of total hip replacement in the United States Medicare population. J Bone Joint Surg Am. 2001;83(11):1622-9.

48. Yasunaga $\mathrm{H}$, Tsuchiya $\mathrm{K}$, Matsuyama $\mathrm{Y}$, Ohe $\mathrm{K}$. High-volume surgeons in regard to reductions in operating time, blood loss, and postoperative complications for total hip arthroplasty. J Orthopaed Sci Off J Jpn Orthopaed Assoc. 2009;14(1):3-9.

49. Bohler C, Weimann P, Alasti F, et al. Rheumatoid arthritis disease activity and the risk of aseptic arthroplasty loosening. Seminars in Arthritis and Rheumatism. 2019.

50. Weldon D. The effects of corticosteroids on bone growth and bone density. Ann Allergy Asthma Immunol. 2009;103(1):3-11.

51. Wooley PH, Schwarz EM. Aseptic loosening. Gene Ther. 2004;11(4):402-7.

52. Mäkelä KT, Matilainen $\mathrm{M}$, Pulkkinen $\mathrm{P}$, et al. Countrywise results of total hip replacement. An analysis of 438,733 hips based on the Nordic Arthroplasty Register Association database. Acta Orthopaed. 2014;85(2):107-16. 
53. Naylor JM, Harmer AR, Heard RC, Harris IA. Patterns of recovery following knee and hip replacement in an Australian cohort. Aust Health Rev. 2009;33(1):124-35.

54. Ozen G, Pedro S, England BR, et al. Risk of serious infection in patients with rheumatoid arthritis treated with biologic versus nonbiologic diseasemodifying antirheumatic drugs. ACR Open Rheumatol. 2019;1(7):424-32.

55. Ramiro S, Sepriano A, Chatzidionysiou K, et al. Safety of synthetic and biological DMARDs: a systematic literature review informing the 2016 update of the EULAR recommendations for management of rheumatoid arthritis. Ann Rheum Dis. 2017;76(6):1101-36.

56. Ito H, Kojima M, Nishida $\mathrm{K}$, et al. Postoperative complications in patients with rheumatoid arthritis using a biological agent: a systematic review and meta-analysis. Mod Rheumatol. 2015;25(5):672-8.

57. Goodman SM, Menon I, Christos PJ, Smethurst R, Bykerk VP. Management of perioperative tumour necrosis factor $\alpha$ inhibitors in rheumatoid arthritis patients undergoing arthroplasty: a systematic review and meta-analysis. Rheumatology (Oxford). 2016;55(3):573-82.

58. Pagani NR, Varady NH, Chen AF, Rajaee SS, Kavolus JJ. Nationwide analysis of lower extremity periprosthetic fractures. J Arthroplast. 2021;36(1): 317-24.

59. Marsland D, Mears SC. A review of periprosthetic femoral fractures associated with total hip arthroplasty. Geriatric Orthopaed Surg Rehabil. 2012;3(3): 107-20.

60. Meek RM, Norwood T, Smith R, Brenkel IJ, Howie CR. The risk of peri-prosthetic fracture after primary and revision total hip and knee replacement. J Bone Joint Surg Br. 2011;93(1):96-101.

61. Schmidt AH, Kyle RF. Periprosthetic fractures of the femur. Orthop Clin North Am. 2002;33(1):143-52, ix.

62. Della Rocca GJ, Leung KS, Pape H-C. Periprosthetic Fractures: Epidemiology and Future Projections. Journal of Orthopaedic Trauma. 2011;25.

63. Lindahl H. Epidemiology of periprosthetic femur fracture around a total hip arthroplasty. Injury. 2007;38(6):651-4.

64. Lewallen DG, Berry DJ. Periprosthetic fracture of the femur after total hip arthroplasty. J Bone Joint Surg Am. 1997;79:1881-90.
65. Miettinen SSA, Törmä SV, Lappalainen JM, Sund R, Kröger $H$. Retrospective population-based cohort study of incidence, complications, and survival of 202 operatively treated periprosthetic femoral fractures. J Arthroplast. 2021;36(7):2591-6.

66. Martins LGG, Garcia FL, Picado CHF. Aseptic loosening rate of the mayo femoral stem with mediumterm follow up. J Arthroplast. 2014;29(11):2122-6.

67. Dayton MR, Incavo SJ. Component loosening in total hip arthroplasty. Semin Arthroplast: JSES. 2005;16(2):161-70.

68. Mulroy WF, Estok DM, Harris WH. Total hip arthroplasty with use of so-called second-generation cementing techniques. A fifteen-year-average follow-up study. J Bone Joint Surg Am Vol 1995;77(12):1845-52.

69. Fender D, Harper WM, Gregg PJ. Outcome of Charnley total hip replacement across a single health region in England. J Bone Jt Surg Br Vol. 1999;81-B(4):577-81.

70. Havelin LI, Vollset SE, Engesæter LB. Revision for aseptic loosening of uncemented cups in 4,352 primary total hip prostheses: a report from the Norwegian Arthroplasty Register. Acta Orthop Scand. 1995;66(6):494-500.

71. Havelin LI, Espehaug B, Vollset SE, Engesaeter LB. Early aseptic loosening of uncemented femoral components in primary total hip replacement. A review based on the Norwegian Arthroplasty Register. J Bone Joint Surg Br Vol 1995;77-B(1): 11-7.

72. Apostu D, Lucaciu O, Berce C, Lucaciu D, Cosma D. Current methods of preventing aseptic loosening and improving osseointegration of titanium implants in cementless total hip arthroplasty: a review. J Int Med Res. 2018;46(6):2104-19.

73. Cherian JJ, Jauregui JJ, Banerjee S, Pierce T, Mont MA. What host factors affect aseptic loosening after THA and TKA? Clin Orthopaed Related Res. 2015;473(8):2700-9.

74. Sundfeldt M, V Carlsson L, B Johansson C, Thomsen P, Gretzer C. Aseptic loosening, not only a question of wear: a review of different theories. Acta Orthopaed. 2006;77(2):177-97.

75. Almutairi K, Nossent J, Preen D, Keen H, Inderjeeth C. The global prevalence of rheumatoid arthritis: a meta-analysis based on a systematic review. Rheumatol Int. 2021;41. 